\title{
How Einstein Did Not Discover
}

\author{
John D. Norton \\ Department of History and Philosophy of Science \\ University of Pittsburgh \\ Pittsburgh PA 15260 \\ www.pitt.edu/ jdnorton
}

\begin{abstract}
What powered Einstein's discoveries? Was it asking naïve questions, stubbornly? Was it a mischievous urge to break rules? Was it the destructive power of operational thinking? It was none of these. Rather, Einstein made his discoveries through lengthy, mundane investigations, pursued with tenacity and discipline. We have been led to think otherwise in part through Einstein's brilliance at recounting in beguilingly simple terms a few brief moments of transcendent insight; and in part through our need to find a simple trick underlying his achievements. These ideas are illustrated with the examples of Einstein's 1905 discoveries of special relativity and the light quantum.
\end{abstract}

\section{Introduction}

How did Einstein make his discoveries? That question has occupied several generations of historians of science. Their efforts have been decisively advanced by Princeton University Press' authoritative, multivolume Collected Papers of Albert Einstein. In collating, annotating, and presenting Einstein's published and unpublished writings in a growing series of volumes, the editors of the project have contributed considerably to primary Einstein scholarship. At the same time, this wealth of material has made it possible for scholars elsewhere to base their work securely in documentary evidence. A recent, introductory distillation of some of this work can be found in the Cambridge Companion to Einstein (Janssen and Lehner, 2014). 
We have learned that the work that led up to Einstein's great discoveries was long and complicated, typically spanning years. There is no easy synopsis that does not mislead in some aspects. Much of the work was devoted to lengthy, mundane investigations. They brought moments of disappointment, frustration and even despair. Einstein persisted with tenacity and discipline. Then there were moments of transcendent insight. However these were rare moments and were only possible because of the painstaking preparatory work.

An armchair theorist can think up many strange ideas that fly in the face of past thinking. Yet Einstein could know that his novel and outrageous thought was the right one of the many possible because of the thoroughness of his preparation. It showed him that nothing else would work. A real understanding of how Einstein discovered requires a fuller appreciation of these lengthy investigations, in all their complexity.

Our efforts to gain this understanding face a formidable obstacle. We want simple answers. We see Einstein perform intellectual wonders like a magician and we want to know the trick. We can learn how a magician materializes a live tiger on stage. The trick, once revealed, does turn out to be simple. We know that there cannot be a comparably simple trick behind Einstein's magic. But we still hope against hope that our necessarily abbreviated accounts still capture what it most important.

If we pay proper attention to the work leading up to the great moments, careful scholarship can be quite successful in explaining how Einstein discovered. However the measured deliberations of cautious scholars are no match for the popular demand for simple answers. Here Einstein's prodigious powers can mislead us. He was wonderfully adept at presenting difficult and hard-won results in engaging and simple narratives, often with compelling and memorable thought experiments. He too felt the popular demand for a simple story and he tried to meet it with cautious simplifications. The broader literature, however, has not generally been so cautious. It all too often meets the same demand with accounts so truncated as to mislead. Worse, the popular demand invites uncontrolled guesses and even opportunistic fabrication.

My goal in this paper is to review and correct some of the more prominent misunderstandings about how Einstein discovered. The mythical literature is so enormous that I cannot hope to penetrate it deeply. However I will try to correct four of the more entrenched myths that have superficial plausibility. This is difficult work since four erroneous 
oversimplification can be stated and propagated in less than the time it take to correct just one. The four discovery myths are indicated below under the section headings

- The Power of Childish Thinking

- The Power of Operational Thinking

- The Power of Breaking Rules

- The Power of a Single Experiment

Their discussion will be interleaved with accounts of two of Einstein's most striking discoveries of 1905 , the special theory of relativity and the light quantum. I will seek to show how an oversimplified account of these discoveries can support the four myths. As a counterpoint, I will try to give an accessible account of how Einstein did make these two discoveries.

We shall see that the bulk of Einstein's work that led to special relativity concerned a problem in electrodynamics: how could the theory be modified to bring it into conformity with the principle of relativity? His efforts over years were a sustained failure and frustration. The idea that a modification to the theory of space and time might solve the problem entered only in the final weeks of years of investigation. It was a desperate, last ditch effort that brought success.

Einstein's discovery of the light quantum did not result from investigations into the wave theory of light. Rather it resulted from work in thermodynamics. It derived from Einstein's facility in inferring from the macroscopic thermodynamic properties of a thermal system to its microscopic constitution. From his work in gas theory and dilute solutions, Einstein knew the distinctive macroscopic imprint of thermal systems composed of many, spatially localized, independent components. He found that signature in high frequency heat radiation and inferred to the light quantum.

Another locus of oversimplified accounts of how Einstein discovered is physics textbooks. For a recent account of how "Physics Textbooks Don't Always Tell the Truth" in relation to the role of experiments in physics, see Franklin (forthcoming). It includes extensive treatment of two experiments discussed below: Millikan's measurements of the photoelectric effect and the Michelson-Morley experiment.

\section{The Power of Childish Thinking}

One of the most tenacious myths about how Einstein discovered is that he relied essentially on adopting a naïve, simple mode of thinking. This mode authorized Einstein to 
discount and ignore all the difficult technicalities of the existing literature, whose distracting complexities, we are to suppose, block progress. The way ahead is to adopt the mode of a precocious child asking childish questions.

Einstein himself seems to have advanced this myth in part through self-deprecating remarks. Here is how one such remark is related, third-hand, by one of Einstein's biographers (Seelig, 1956, pp. 70-71): ${ }^{1}$

One of Einstein's typical remarks was reported to me from Falmouth, Massachusetts by James Franck ... "When I asked myself," Einstein said to Franck, "how it happened that I in particular discovered the relativity theory, it seemed to lie in the following circumstance. The normal adult never bothers his head about space-time problems. Everything there is to be thought about it, in his opinion, has already been done in early childhood. I, on the contrary, developed so slowly that I only began to wonder about space and time when I was already grown up. In consequence I probed deeper into the problem than an ordinary child would have done."

This report is from a winner of the Nobel Prize in physics, James Franck. A no lesser authority in relativity theory, John Archibald Wheeler, gives a similar assessment of the source of Einstein's creative powers. (Wheeler, 1980, p. 102):

Search out the simple central principles of this physical world - that was becoming Einstein's goal. But how? Many a man in the street thinks of Einstein as a man who could only make headway in his work by dint of pages of complicated mathematics; the truth is the direct opposite. As Hilbert put it, "Every boy in the streets of our mathematical Gottingen understands more about four-dimensional geometry than Einstein. Yet, despite that, Einstein did the work and not the mathematicians."[Footnote*] Time and again, in the photoelectric effect, in relativity, in gravitation, the amateur grasped the simple point that had eluded the

1 This remark is elsewhere (Fölsing, 1997, p. 13,p. 743) reported as written "in a letter" to Franck by Einstein. The citation (p. 743) gives no date for the letter and is to another of Seelig's works. I have not located a letter from Einstein to Franck with this content. 
expert. Where did Einstein acquire this ability to sift the essential from the nonessential?

Wheeler gives two related ideas. Einstein's power depends on his being an "amateur," so he could see things that "eluded the experts." Second, Wheeler deprecates "pages of complicated mathematics." That is not how Einstein made his discoveries. ${ }^{2}$

In support, Wheeler invokes the authority of David Hilbert, who was, in Einstein's time, a dominant figure in mathematics and made significant contributions to the mathematics of relativity theory. The footnote reference for his quote from Hilbert is to an unsourced quote in Philipp Frank's biography. ${ }^{3}$ Frank (1947, p. 206) continues with another, comparable, unsourced quote from Hilbert:

And he once asked a gathering of mathematicians: "Do you know why Einstein said the most original and profound things about space and time that have been said in our generation? Because he had learned nothing about all the philosophy and mathematics of time and space."

Einstein's ignorance is not just of the pertinent mathematics, but of the philosophy as well. ${ }^{4}$

Philipp Frank was not just a biographer but also a colleague of Einstein's. When Einstein left his professorship in Prague, Frank was his successor. So could report with some insight.

2 One can only guess how such misperceptions arise. I have spent many, long hours working through Einstein's complicated mathematical computations in his Zurich notebook, which contains the computations made in preparation for general relativity.

3 Wheeler footnotes a German edition, but his text gives the quote from the English translation (Frank, 1947, p. 206)

${ }^{4}$ It is plausible that Frank was reporting correctly what Hilbert said. However context matters and it is likely that Hilbert intended something narrow in his remarks. Einstein had been very slow to adopt and even disparaging of the four-dimensional methods of spacetime geometry introduced by Hilbert's Goettingen colleague, Hermann Minkowski. These methods were, presumably, something well-known to "every boy in the streets of our mathematical Gottingen." Hilbert's own philosophical inclinations leant towards Immanuel Kant. Einstein dismissed the Kantian approach and, in his earlier years, favored a positivistic philosophy such as advanced by Ernst Mach. A Kantian in this time would be obliged to find that outlook naïve. 
Elsewhere in his biography (p. 112), Frank reports of Einstein's participation in the weekly physics colloquium in Berlin. Once again, the power of Einstein's naivete is the driving theme:

On such occasions there are always many who are ashamed to ask questions because they do not wish to appear ignorant, and usually it is just the people who take the longest to comprehend something who are shy. Since Einstein could never be suspected of slow comprehending, he did not hesitate to ask questions that would otherwise be considered as naïve. Such 'naïve' questions, however, are often very stimulating, because they frequently deal with fundamental problems that no one really dares to touch. Most specialists would like to make believe that they understand the fundamentals and are only seeking to explain secondary matters. Einstein's questions, which very often threw doubt upon a principle that appeared self-evident, gave the seminar a special attraction.

The image of Einstein the childish naïf proved irresistible. Childish innocence is the key to great discovery. How could we not be charmed by such a story? How irresistible must it be to every outsider who finds the ponderous content of physics overwhelming? Such outsiders are now reassured by the example of Einstein that they can just ignore all the fancy details. Just ask precocious, childish questions that annoy the experts and you are on the right track. Yes, you too can be the next Einstein and you do not have to know much to do it.

\section{The Discovery of Special Relativity}

These are all testimonial to the power of Einstein's supposed naivete. Is there direct evidence of it in Einstein's work of its power? The natural place to look is Einstein's discovery of special relativity. It has some of his most famous thought experiments involving beguilingly simple arrangements of light signals and clocks. The much-celebrated 1905 "On the electrodynamics of moving bodies" was published in one of the most prestigious physics journals of the German academic establishment of the time, Annalen der Physik. We find in its first section a remark that could be used to instruct a six-year-old child:

If, for instance, I say, "That train arrives here at 7 o'clock," I mean something like this: "The pointing of the small hand of my watch to 7 and the arrival of the train are simultaneous events." 
The innocence of the remark is misleading. What led to it were many years of labor. Einstein himself recalled in 1952 "the seven and more years that the development of the Special Theory of Relativity had been my entire life." 5 While much of the details of these seven and more years is lost, it is possible to reconstruct some major foci and turning points, as I have tried to do in my Norton (2004).

To summarize briefly the technically intricate account developed in Norton (2004), the most important observation is that the great bulk of this time was spent grappling with problems in electrodynamics that now seem quite arcane and involve no modifications to our notions of space and time. The major focus was the existence of an ether state of rest in electrodynamical theories of the time. A youthful Einstein in 1895, at the age of sixteen, had conceived an experiment that would detect the earth's motion with respect to this state of rest. By the end of 1901, Einstein had become convinced that the phenomena of electrodynamics did not support the existence of such a state of rest.

The task Einstein now faced was to reconfigure electrodynamical theory so that it no longer needed an ether state of rest. That is, the theory was to be brought into conformity with the principle of relativity, which requires an equivalence of all inertial states of motion. This was a daunting task. Maxwell-Lorentz electrodynamics was one of the great achievements of nineteenth century physics. It had taken a wealth of experimental results in electricity, magnetism and light and captured them all in one set of differential equations, now known as "Maxwell's equations." The fit was close and precise. The slightest tinkering with the theory almost always creates a conflict with one or other of the experimental results.

The problem occupied Einstein for years. From later recollections we know that he came very close to a conservative solution that required no novel notions of space and time. One version of electrodynamical theory uses so-called retarded potentials: the fields at a point in space are recovered by adding up the field producing effects of all sources elsewhere and at earlier times. The earlier time chosen, the so-called retardation time, is fixed by assuming that electrodynamical effects propagate at the constant $\mathrm{c}$, the speed of light, with respect to the ether. A small adjustment, later exploited by Ritz, shows great promise of bringing conformity with the principle of relativity. One merely adjusts the retardation time so that the electrodynamical

\footnotetext{
5 A quoted in Norton, 2003,p. 49.
} 
effects propagate at $\mathrm{c}$ with respect to the source. One can otherwise keep the basic formulae unchanged. The ether state of rest is written out of the theory and it looks as if all its experimental successes may be preserved.

These efforts are normally recalled as efforts to find an emission theory of light. In such a theory, the speed of light is fixed at $\mathrm{c}$ with respect to the emitter. Here it differs from how light behaves in the then standard theory, in which light propagates at $\mathrm{c}$ with respect to the ether. That means the light coming from the headlights of a rapidly moving car travels at more than c with respect to the road, but the light from the tail lights moves at less than c. If Einstein were to succeed in conforming electrodynamics to the principle relativity, an automatic consequence would be that light waves would propagate in accord with the emission theory. To characterize Einstein's task merely as finding an emission theory of light woefully understates Einstein's task. The real and bigger task was to bring all the other effects of electrodynamics into conformity with the principle of relativity.

Einstein may have come close with this theorizing. However, he never achieved complete conformity. While realizing an emission theory of light was not sufficient, it was necessary. Einstein's later recollections are rich in reasons for the failure of an emission theory of light to conform with the fuller electrodynamical theory. These are traces of much thought and labor spent on the problem.

It turned out, however, that a solution to his problem lay in a different direction and was close at hand. Einstein did not need to alter electrodynamical theory at all to bring it into conformity with the principle of relativity. He could leave it untouched. All he had to do was alter default ideas about how things change when we move between different states of motion.

Early in his explorations, Einstein had already found parts of this solution, which was recounted in the opening paragraph of his 1905 paper as the "magnet and conductor" thought experiment. An ether-based electrodynamics seems to provide a simple means of checking whether a system is at rest in the ether or moving through it. A magnet at rest in the ether is surrounded merely by its own magnetic field. If the magnet moves in the ether, then the magnetic field at some fixed point in the ether will change with time. These changes induce a new entity, an electric field. The existence of this induced electric field is, it would seem, a distinctive marker of the magnet's motion through the ether. An observer at rest in the ether can detect it in 
the space around a moving magnet merely by measuring the electric current it induces in an electrical conductor as the magnet moves past.

The trouble, Einstein noted in his thought experiment, is that attempts to detect this distinctive marker are confounded by a second effect. If it is not the magnet but the observer and conductor that moves through the ether, then the mere fact of moving the conductor through the magnetic field of the magnet is sufficient to generate an electric current. That current turns out to be exactly the same in magnitude as in the case of the magnet in motion but the observer and conductor at rest. Here was a familiar circumstance. The ether theory insists that there is a factual difference between the case of the magnet at rest in the ether and the magnet moving through it. However observation fails to detect it.

This failure, Einstein later recalled, had both convinced him of the correctness of the principle of relativity and, in slightly more modern terms, that electromagnetic field quantities would transform in novel ways between inertial frames of reference. A magnetic field without electric field, for example, will have an added electric field component if it is viewed from another frame of reference.

The mathematical apparatus needed to complete this approach had already been developed by H. A. Lorentz in his theorem of corresponding states. Einstein knew well an earlier version of the theorem from Lorentz's work of 1895. The difficulty of pursuing the approach is that a full implementation requires that more than field quantities transform in unexpected ways. Spaces and times must do so as well. Lorentz introduced an auxiliary time variable, his "local time," in each inertial frame of reference. To complete the approach, Einstein would have to accept that this local time was just the time simpliciter of the inertial frame of reference. That would in turn require Einstein to accept the odd result that judgments of simultaneity would differ according to the frame of reference.

Once Einstein accepted ${ }^{6}$ this result of the relativity of simultaneity, all his problems were solved. He could retain Maxwell's equations unchanged and with them all their many

${ }^{6}$ Einstein remarked on the importance of the experimental effects of stellar aberration and Fizeau's measurements of the speed of light in moving water in his discovery of special relativity. I have conjectured in Norton $(2004, \S 7)$ that their importance lay in them being experimental manifestations of the relativity of simultaneity. 
experimental successes. He had no further need of the troublesome emission theory light. The speed of light would remain a universal constant $\mathrm{c}$, the same value in each inertial frame and independent of the motion of the emitter. This conclusion would be elevated in Einstein's 1905 paper to axiomatic status as the "light postulate." In an introductory historical preamble to a 1907 survey of relativity theory, Einstein remarks in words that to me have an autobiographical ring (Einstein, 1907, p. 413):

One needed only to realize that an auxiliary quantity that was introduced by H. A.

Lorentz and that he called "local time" can simply be defined as "time."

With that insight, Einstein had the complete solution.

All that remained was the task of formulating the solution in an accessible manner. The first step was to separate out the new transformation properties of space and time as a distinct theory, expressible independently of electrodynamical theory. This became the first "kinematical part" of Einstein's 1905. Somehow Einstein had to make the odd but key result of the relativity of simultaneity palatable. That was achieved with a famous thought experiments in the first sections of the paper (to be discussed further below). Einstein showed that if one synchronized clocks by means of light signals, the relativity of simultaneity followed.

\section{The Truncated Story}

How did Einstein discover special relativity? The real story lies in the seven and more years of struggle with the problems in electrodynamics just recounted. That is what one must study if one wants to know how Einstein did it. Alas, this story is technically complicated and all attempts to tell it are hampered by a dearth of sources. Worse, the story does not admit the sort of simple encapsulation that we associate with Einstein. Instead of grappling with these difficult details, the story is commonly truncated merely by recalling the vivid and simple thought experiments Einstein provided us.

In advance of 2005, the centenary of Einstein's 1905 discovery was celebrated throughout the media. Here is how Discover magazine recounted Einstein's discovery in their Special Einstein Issue:

Einstein used what were called thought experiments to achieve his radical simplifications. Special relativity emerged from a line of thinking that began when Einstein asked what would happen if he could chase a ray of light. His breakthrough 
realization of the relativity of time turned on a series of mental cartoons featuring trains and clocks.

The entire story is reduced to Einstein's use of thought experiments. Two are alluded to, one from the very start of Einstein's investigations and one from their conclusion. What is completely omitted is the real work of Einstein's investigations into electrodynamical theory. This is a widespread omission and responsible for much misunderstanding. Einstein had an almost magical power to distill complex physics into simple but powerful thought experiments. Take those thought experiments in isolation and one develops the notion that Einstein's discovery was merely the result of childish play with ideas, "mental cartoons featuring trains and clocks."

Let us review the place of these thought experiments in Einstein's discovery.

\section{Einstein Chasing a Beam of Light}

The first thought experiment alluded to above was coaxed retrospectively out of an obliging Einstein. While is it beloved by commentators, I will argue below that it is unclear how much we can learn of Einstein's discovery process from it. It is as much a reflection of the simple cartoon image people would like of Einstein's discovery process, as it is of the real events. First, let us recall what we know of the thought experiment.

In 1946, an aging Einstein set out his autobiographical reflections on his life in science. He recounted reflections from over half a century before that led to the special theory of relativity. He had sought, he recalled, a universal principle analogous to the laws of thermodynamics. He continued: (Einstein, 1949, pp. 52-53/ pp. 49-50):

How, then, could such a universal principle be found? After ten years of reflection such a principle resulted from a paradox upon which I had already hit at the age of sixteen: If I pursue a beam of light with the velocity c (velocity of light in a vacuum), I should observe such a beam of light as an electromagnetic field at rest though spatially oscillating. There seems to be no such thing, however, neither on the basis of experience nor according to Maxwell's equations. From the very beginning it appeared to me intuitively clear that, judged from the standpoint of such an observer, everything would have to happen according to the same laws as for an observer who, relative to the earth, was at rest. For how should the first 
observer know or be able to determine, that he is in a state of fast uniform motion?

One sees in this paradox the germ of the special relativity theory is already contained.

The tone is much less certain in another account of the same thought experiment included in reminiscences of his student year at the gymnasium in Aarau. Einstein (1956, p.10) recalled:

During this year in Aarau the following question came to me: if one chases a light wave with the speed of light, then one would have before one a time independent wave field. But such a thing appears not to exist! This was the first child-like [German: kindliche] thought experiment related to the special theory of relativity. Discovery is not a work of logical thought, even if the final product is bound in logical form.

\section{The Naïve Thought}

This thought experiment has proven especially appealing to those who locate Einstein's creative powers in childish thinking. Einstein's chasing a light beam thought experiment is identified by Craig Loehle in his Becoming a Successful Scientist: Strategic Thinking for Scientific Discovery. This might not be a work we would take too seriously, were it not published by a premiere academic press, Cambridge University Press. Loehle (2010, pp. 30-31), in a chapter section on "Releasing Creativity," reports of this thought experiment:

Those whom we note as outstandingly creative have often been described as possessing a childlike innocence or sense of wonder, and they ask seemingly naïve questions. Einstein asking what would happen if you rode on a rocket at the speed of light and looked at a mirror held in front of you (he concluded that you would not be able to see your reflection), sounds like the ultimate naïve question, like the silly questions kids ask, but it turns out to be on with profound consequences. This attitude contributes to creativity by keeping the mind flexible.

A comparison of Loehle's text with Einstein's words shows that this literature feels no obligation to report accurately. There is no talk of mirrors or reflections in Einstein's text. A paragraph later, the synoptic moral comes:

A major obstacle to discovery is not ignorance but knowledge. 
This gets it exactly backwards in the case of Einstein. He succeeded precisely because he knew so much and thus could find the elusive right way forward.

A loose attitude to sources seems quite common in the literature that seeks to draw morals about discovery from Einstein's thought experiment. Howard Gardner is the John H. and Elisabeth A. Hobbs Professor of Cognition and Education at the Harvard Graduate School of Education, a MacArthur Fellow and author of Frames of Mind: The Theory of Multiple Intelligences. This work has enjoyed multiple printings since its first release in 1983. There, Gardner (2011, p. 157) advances the general claim:

It is left to the greatest scientists to pose questions that no one has posed before, and then to arrive at an answer that changes for all time the way in which scientists (and eventually laymen) construe the universe.

He immediately illustrated this claim with Einstein's example:

Einstein's genius lay in his persistent questioning of the absoluteness of time and space. Already as a teenager, Einstein pondered what our experience would be like if we ourselves were operating from the point of view of light, or, to put it more concretely, if we rode on a beam of light. Suppose, he asked, that we were looking at a clock, but were flying away from it at the speed of light. The time on the clock would then be frozen because a new hour could never travel fast enough to catch up with us; on the beam of light, the time of that clock would remain perpetually the same.

This recounting scarcely resembles Einstein's own brief account. Someone versed in relativity theory will identify the account as a novice misunderstanding of how the relativistic slowing of clocks arises and quite unworthy of attribution to Einstein. The unfettered imaginings in Gardner's text of Einstein's thought continues, unfortunately, for several paragraphs more.

\section{Behind Einstein's Chasing a Light Beam Thought Experiment}

These cartoonish impersonations of Einstein's thought experiment are possible because Einstein's account of the thought experiment is brief, cryptic and puzzling.

First, the events recounted happened in late 1895 or early 1896. Yet Einstein mentions Maxwell's equations, the key equations of the 19th century electrodynamics. He did not learn them until his university studies around 1898. Einstein's first report of the thought experiment in 
his own writings comes in 1946. The thought experiment does not appear in the 1905 special relativity paper, in any later writings prior to 1946 or in correspondence.

Second, unlike the luminous clarity of Einstein's other thought experiments, it is not at all clear how this thought experiment works. In the dominant theories of the late nineteenth century, light propagates as a wave in a medium, the luminiferous ether. It was an entirely uncontroversial result in the theory that, in a frame of reference that moved with the light, the wave would be static. There is no reason for us to be puzzled. We do not see frozen light since we are not moving at the speed of light through the ether.

Elsewhere (Norton, 2004, §5; 2013) I have put some effort into understanding both the thought experiment's content and how the thought experiment came about. The first written evidence of it comes from the gestalt psychologist Max Wertheimer's report in a chapter of his Productive Thinking. Wertheimer, a gestalt psychologist, had interviewed Einstein in 1916 and possibly later concerning his discovery process for special relativity. The product was an account that went through ten "acts," like a play, each later and of increasing sophistication. The recounting of Einstein's youthful imagining of the chasing of a light beam comes in the first act.

“These were wonderful days," Wertheimer reported (1959, p. 213), "beginning in 1916, when for hours and hours I was fortunate enough to sit with Einstein, alone in his study, and hear from him the story..." And then: "During these long discussions I questioned Einstein in great detail about the concrete events in his thought..." Cleary, we cannot assume that the story was narrated spontaneously with Wertheimer listening passively. Rather the account that Wertheimer eventually relates has been coaxed out of Einstein by Wertheimer, who questioned him "in great detail."

What Wertheimer prompted from Einstein was quite hesitant and inconclusive. The text is too long to quote here. But it is replete with remarks like (p. 169) "The process started in a way that was not very clear, and is therefore difficult to describe-in a certain state of puzzlement." and (p. 170) "Back of all of this there had to be something that was not yet grasped, not yet understood. Uneasiness about this characterized young Einstein's state of mind at the time."

We have nothing in writing from Einstein himself until his drafting of this “Autobiographical Notes" in 1946. Why does it appear there? I have conjectured $(2004, \S 5)$ that it was prompted by Max Wertheimer. He delayed publishing his account of Einstein's thought 
until his Productive Thinking, published posthumously in 1945, after his death in 1943. In that final year of his life, Wertheimer had sent Einstein drafts of the chapter for his comments and Einstein had responded. Thus it seems likely that Einstein included the thought experiment after his recollections were prompted and possibly reconfigured by Wertheimer's account. This makes Einstein's text challenging to interpret. It may well be a kind of recovered memory.

The puzzling centrality of Maxwell's equations in Einstein's account of the thought experiment is readily explained if we allow that the thought experiment persisted for a few years in Einstein's thought, while he worked towards the special theory of relativity. In my reconstruction, the thought experiment is opaque only if we relate it to ether theories of light. We saw that, in the years leading up to special relativity, Einstein put considerable effort into developing an emission theory of light. Einstein's chasing a light beam thought experiment becomes intelligible and important if we regard it as encapsulating some of Einstein's many objections to the emission theory of light, when coupled with the principle of relativity. The details are given in Norton $(2004, \S 5 ; 2013)$.

One can quickly see that the fit to the emission theory is natural by imagining that we move at $\mathrm{c}$ with the light away from its source. If the principle of relativity holds, that is equivalent to having a source receding from us at $\mathrm{c}$. Then the emission theory requires that the speed of the light relative to us is $\mathrm{c}-($ speed of source $)=\mathrm{c}-\mathrm{c}=0$. We would find a frozen light wave in our frame of reference. Since the principle of relativity holds, this occurrence could happen in any inertial frame of reference, including the one we occupy now. All we need is for there to be one source of light moving at $\mathrm{c}$ in our frame for that source to paint a frozen light wave across space. That there might be such a source somewhere is not far-fetched, so we might well expect to see frozen light. However, we do not. That is unexpected. Einstein's remark is now quite to the point.

What does this leave us? Einstein's reports of the thought experiment are a mixture of parts, pulled together by Einstein much later in life. It came, I believe, at the prompting of reading Wertheimer's account. The original story was coaxed out of Einstein by an eager Wertheimer. His hours and hours of interrogation were not those of a neutral questioner, but of a psychologist with a definite view. He needed a simple story he could relate to non-technical readers. Perhaps Einstein, in the years prior to his public celebrity, was flattered by the attention that a scientist of the mind was giving to his mind. Might he struggle to oblige with a good story? 
Might he plumb for some fragmentary memory of some early experience that Wertheimer could fit into the narrative he was subtly pressing on Einstein? That earlier experience might not have been of much consequence, but it could grow in importance with Wertheimer's encouragement. Even under pressure from an eager Wertheimer, the initial imaginings of the sixteen-year-old Einstein produced nothing but a state of puzzlement or uneasiness. That gave the older Einstein of his Autobiographical Notes little to report. So it was easy for Einstein to let the narrative mutate into a later episode in which the thought of chasing a light beam did produce a useful result: it encapsulated in a simplified way his long struggle with the emission theory of light and its eventual rejection.

We cannot derive from this story any support for the idea that naïve and childish thinking had a significant role in Einstein's discovery process. At best we can learn that Einstein found that picture welcomed by his readers and interlocutors and that he obliged by delivering it.

\section{Clocks, Rods, Light Signals and Simultaneity}

The second set of thought experiments associated with Einstein's discovery of special relativity appears first in Sections 1 and 2 of Einstein's 1905 “On the electrodynamics of moving bodies." In Section 1, he asserts that we cannot know if two clocks at rest at different places A and B in space are properly synchronized. He proposes an operational definition employing light signals for affirming it. In simplified form, if a light signal leaves clock A when it reads zero, is reflected at clock $B$ when it reads one and returns to clock A when it reads two, then the two clocks are properly synchronized. Einstein follows up in Section 2 by considering an observer in motion with respect to the clocks. That observer, Einstein shows, will not judge the clocks to be properly synchronized. A similar result follow for the timing of events. While one observer may judge two spatially separated events to be simultaneous, a second observer in relative motion to the first may not. This is the "relativity of simultaneity."

These are some of Einstein's best-known thought experiments. Another more familiar development of essentially the same thought experiments arises in Einstein's 1917 popular work (Einstein, 1917, Ch. IX-X). The abstract moving and stationary coordinate systems are replaced by a train moving along an embankment and events appear as lightning strikes.

For our purposes, it essential to see how Einstein's thought experiments on clock synchronization deliver their results. That delivery depends entirely on the theoretical 
assumptions employed. If the thought experiments are carried out within standard theories of Einstein's era, then no unusual results follow.

Take an ether-based electrodynamics, in which light propagates at $\mathrm{c}$ with respect to the ether. If the A and B clocks are at rest in the ether, the definition will correctly synchronize the clocks in accordance with the time that enters into the ether-based Maxwell equations. If, however, the A and B clocks are moving in the ether, then the speed of light in the two directions from $\mathrm{A}$ to $\mathrm{B}$ and back from $\mathrm{B}$ to $\mathrm{A}$ will be different. As a result, the definition will fail to synchronize the clocks properly by the standards of the ether-based theory. There is no mystery in this failure. Definitions can fail to conform with what else we know. In a fit of perversity, we might define clocks to be synchronized if they have the same weight. That silly definition would be incompatible with we otherwise know about time and clocks.

What if we wish to retain the principle of relativity so that our synchronization procedure must work in all inertial frames of reference? We must now move from an ether-based electrodynamics, for such a theory contradicts the principle of relativity. The principle of relativity is satisfied by a Newtonian, emission theory of light: the speed of emission of light $\mathrm{c}$ is boosted by the speed $\mathrm{v}$ of the emitter. If the $\mathrm{A}$ and $\mathrm{B}$ clocks are moving at $\mathrm{v}$ in a direction from $\mathrm{A}$ to $\mathrm{B}$, then light will move at speed $\mathrm{c}+\mathrm{v}$ from $\mathrm{A}$ to $\mathrm{B}$ and speed $\mathrm{c}-\mathrm{v}$ from $\mathrm{B}$ to $\mathrm{A}$. The effect of these differing speeds is that all inertially moving observers will agree that the two clocks have been properly synchronized. Once again there are no unusual results. There is no relativity of simultaneity.

To recover the relativity of simultaneity, Einstein adopts elements from each of these two settings. From the Newtonian setting, Einstein takes the principle of relativity and from 19th century electrodynamics he takes the idea that light propagates at a definite speed, $\mathrm{c}$, which he now calls the "light postulate." These principles are, to use Einstein's words, "apparently irreconcilable."

The point of Einstein's thought experiments is to show that the two principles can be reconciled, if we adopt the relativity of simultaneity. The observer in the frame of reference of the clocks synchronizes the clocks with the tacit assumption that light, traveling at c, takes the same time to traverse both directions, A to B and back to A. An observer in another frame of reference retains the assumption that light travels at c. Since the clocks are moving, for example in direction $\mathrm{A}$ to $\mathrm{B}$, the light will take longer to move from $\mathrm{A}$ to $\mathrm{B}$ than from $\mathrm{B}$ to $\mathrm{A}$. This second 
observer will infer that the first observer has not synchronized the clocks correctly. That difference of judgment is the relativity of simultaneity. No further contradiction ensues.

The operational analysis of simultaneity is, by itself, quite inert. In standard theoretical contexts, it produces nothing untoward. To get non-standard results, one must employ the apparently irreconcilable principle of relativity and the light postulate. Einstein is quite clear about this. He begins his (1905) Section 2, "On the Relativity of Lengths and Times," by asserting: "The following reflexions are based on the principle of relativity and on the principle of the constancy of the velocity of light." The thought experiment serves the expository purpose of reassuring his readers of the compatibility of the two principles, but that this compatibility requires the relativity of simultaneity.

Conversely, one does not need Einstein's clock synchronization procedure to arrive at the relativity of simultaneity. As textbooks on relativity theory have for generations, one merely derives the Lorentz transformation by finding which linear transformation of the coordinates of space and time preserve the speed of light at $\mathrm{c}$. The relativity of simultaneity is then read directly from the Lorentz transformation.

\section{The Power of Operational Thinking}

Einstein's thought experiments on clock synchronization comprise, in my view, what might well be the single most important conceptual analysis of the twentieth century, when measured by its influence. Percy Bridgman's (1927) polemical manifesto of the doctrine of operationism begins on page one with a tribute to Einstein and avers that a study of what Einstein did will ensure (p.2) "that another change in our attitude, such as that due to Einstein, shall be forever impossible." He proceeds to a detailed development of Einstein's operational definitions of length and of time. Hans Reichenbach's (1957) influential treatise on the philosophy of space and time made Einstein's thought experiments central to the analysis of time. Einstein had described his clock synchronization procedure as providing a "Definition of Simultaneity," which was the title of relevant section of his 1905 paper. Reichenbach stressed that the procedure was chosen conventionally, so that judgments of simultaneity within one reference frame are merely conventions. This thesis of the conventionality of simultaneity became a major locus of philosophical dispute in the remainder of the century. 
There are many more examples. Intellectuals of the eighteenth century enlightenment sought to mimick Newton's methods in order to solve new problems. So many physicists of the twentieth century repeatedly turned to operational analyses embedded in suggestive thought experiments, all modeled on Einstein's 1905 example.

Since we are now so powerfully taken by these thought experiments, it is but a small step to attribute them an outsized role in Einstein's process of discovery. The myth of the power of childish thinking in Einstein's work now merges into a second myth. The key to the discovery of special relativity, the myth asserts, was demanding operational answers to questions that we otherwise would think beyond the need of scrutiny. What does is mean to say that two events are simultaneous? What does it REALLY mean to say this?

Here is a version of the new myth in Mook and Vargish (1987, p. 54). They are tempted once again to imagine Einstein's process as childlike.

Albert Einstein's work on relativity theory was guided by a series of simple even childlike questions. He pursued the answers to these questions remorselessly, and in answering them he began to demolish the Newtonian foundations of physical science. His questions were fundamental: they were questions of definitionindeed, definitions of some of the basic terms used by scientists and others to describe the world, terms such as "space" or "time."

Einstein sought definitions that contained implicit instructions for making physical measurements in order to determine the meaning of the terms; that is, his definitions were so-called “operational definitions,"...

Similarly, Kamlah (1994, p. 174), reports Einstein's achievement in his 1905 special relativity paper as:

He started his considerations by formulating a definition of simultaneity, a concept which nobody had defined until then. Einstein proved that the introduction of new physical concepts by operational definitions can lead to a scientific revolution. The new definition led to the theory of special relativity[7]... Thus the definition of simultaneity was not so much the result of an analysis of already accepted theories

7 Words omitted in ellipses: "which was actually a metatheory, a set of fundamental principles forming the frame and precondition of all different physical theories." 
but rather a creative process of designing new theories, an essential innovation in physics.

This appraisal gets the process backwards. Einstein's years-long investigations in electrodynamics pressed him to accept the principle of relativity, the light postulate and, consequently, the relativity of simultaneity. That is the essence of it. The operational analysis of the thought experiments functioned only to reassure his readers and, perhaps Einstein himself, that this consequence is acceptable. The operational analysis by itself is quite inert and, without Einstein's two principles, produces nothing novel.

A similar misappraisal invited by Galison's (2003) work, which shows that Einstein's idea of synchronizing clocks by light signals was not an idiosyncratic invention by Einstein. Rather the synchronization of well-separated clocks by electric signals had become a major project in Einstein's time, essential to the running of a high speed rail system and leading to the establishment of national and international time zones. Patents for synchronization systems passed through the Bern patent office during Einstein's employment there as a patent examiner.

The difficulty is to decide what significance these facts have. In answering, Galison (2003, p. 36-37, his emphasis) repeats the now familiar idea of naïve questions leading to momentous results:

What time do the map makers make it out to be in Berlin when it is noon in Paris? What time is it down the line when the train pulls into Bern? In posing such questions, Poincaré and Einstein seem, at first glance, to be asking questions of stunning simplicity. As was their answer: two distant events are simultaneous if coordinated clocks at the two locations read the same-noon in Paris, noon in Berlin. Such judgments were inevitably conventions of procedure and rule: to ask about simultaneity was to ask about coordinated clocks. Their proposal: Send an electromagnetic signal from one clock to the other, taking into account the time the signal takes to arrive (as approximately the speed of light). A simple idea of breathtaking consequences for concepts of space and time, for the new relativity theory, for modern physics, for the philosophy of conventionalism, for a worldcovering network of electronic navigation, for our very model of secure scientific knowledge. 
Alas, the claim is quite mistaken. The idea that we synchronize clocks by electromagnetic signals has no breathtaking consequences for space and time, relativity theory, modern physics, philosophy or our model of scientific theories. As we just saw, those consequences come from Einstein's two postulates and his hard-won recognition that both are to be maintained. Without them, none of these last breathtaking consequences follow from the synchronization procedure. With them, the consequences will follow, whatever mode of reasoning we choose. An operational analysis is merely a convenient way to arrive at them.

Galison's account of the deeper significance of the many facts he has collected is opaque to me. ${ }^{8}$ He disavows the strong causal theses. "Philosophical and physical reflections," he says, "did not cause the deployment of coordinated train and telegraph time." (p. 39, Galison's emphasis) Then: "Nor did the vast networks of electro-coordinated clocks of the of the late nineteenth century cause or force the philosophers or physicists to adopt the new convention of simultaneity." Instead it is "a slice through layers of physics, technology, and philosophy that cuts high and low, an exploration of synchronized clocks crisscrossing back and forth between the wiring of the oceans to marching Prussian armies ..." (p. 37) Galison offers a new "metaphor" of "critical opalescence" with "wild fluctuations back and forth between the abstract and the concrete..." (p. 40)

The metaphor appears to me as an overheated description of the banality that things in the world are connected and intersect in many ways. Einstein was not an automaton of the intellect generating theories. He was a man who ate sausage and soup, suffered stomach ailments, warmed himself by infrared radiation in front of a fire, abhorred militarism and sat in trains drawn by the heat engines of massive locomotives. Whether any of these many intersections were significant is a matter of investigation and evidence. The story of clock synchronization technology is, I am pleased to say, hugely entertaining. But I can find no point of illumination of special significance for Einstein's discovery.

Another familiar technology was rail travel. Einstein traveled often on trains. These trains appear as surrogates for inertial frames of reference in his thought experiments. I doubt anyone would regard the existence and use of trains in Einstein's time as especially illuminating for his discovery of special relativity. Everyone traveled on trains, yet only Einstein discovered

${ }^{8}$ For a lengthier appraisal, see Martinez (2004). 
relativity. The same is true of electromagnetic clock synchronization technology. The more we learn that it was a commonplace of the science and technology of the time, the less it is distinctive of Einstein's discovery process. Everyone knew about it, it seems, yet only Einstein discovered special relativity.

While Galison disavows the crude claims of causal influence, the reception of his work has embraced them. I am not alone in my failure to grasp the critical opalescence metaphor. Here is how the Stanford Report of May 16, 2005, announced the coming Hofstadter lecture by Galison (Madden, 2005):

Galison refutes the myth that Albert Einstein and Henri Poincaré were solitary thinkers and emphasizes the great influence of the social, political and technological climate on the development of scientific theory. Relativity, for example, may have been suggested to Einstein from train station clocks.

\section{The Power of Breaking Rules}

Einstein's discoveries ended up breaking with the old physics. He also harbored a longstanding contempt for authority. "To punish me for my contempt of authority," he said in an aphorism for a friend, "Fate has made me an authority myself."' A young Einstein in 1901 complained of the stubbornness of the well-established physicist Drude, who had brushed off strong objections to his work from Einstein by remarking that a colleague agrees with Drude. In frustration Einstein wrote to a correspondent: "Blind obedience to authority is the greatest enemy of the truth." 10

It is a small step from these facts to the myth that Einstein's successes were powered by a contrarian streak that set out to break the rules. This is a familiar theme in the popular literature. Thorpe's (2011) self-improvement guide is entitled optimistically How to Think Like Einstein: Simple Ways to Break the Rules and Discover your Hidden Genius. Chapter 1, "Einstein's Secret," gets right to it: “...one universal principle is at the core of learning to think like a genius: you've got to break the rules." (boldface in original)

\footnotetext{
9 As quoted in Calaprice (2011, p. 12), dated September 18, 1930.

10 Einstein to Jost Winteler, July 8, 1901. Doc. 115 in Stachel (1989). Translation, Calaprice (2011, p.161).
} 
I will argue otherwise below in the case of Einstein's discovery of the light quantum: whatever contrarian streak may have been in his work, his success depended far more on the thoroughness of his investigations. His contrarian impulses were subordinate to his need for good reasons. This manifests clearly in his later rejection of modern quantum theory. Einstein could not abide its rule-breaking indeterminism and non-locality. He sought refuge in the comfortable, traditional confines of his unified field theory.

\section{The Power of a Single Experiment}

The example of Einstein's discovery of the light quantum will illustrate another popular myth about what powered Einstein's discoveries. There is, in each case, a single, perplexing, powerful, decisive, crucial experiment. Only Einstein, it is said, was able to interpret the experiment correctly and arrive at a great discovery.

This myth is best known through the example of the Michelson-Morley experiment. Contrary to many reports, Einstein did not formulate the theory as a direct response to its null outcome. The mistake is an easy one to make. It was long standard for pedagogic accounts of special relativity to begin with an account of the experiment and jump directly to special relativity. The pattern starts with Einstein's (1907) early review. It introduces the MichelsonMorley experiment and no others in its opening pages. Holton's (1969) analysis of the myth is standard and includes numerous examples. To it, we should add that the null result of the Michelson-Morley experiment was unhelpful and possibly counter-productive in Einstein's investigations of an emission theory of light, for the null result is predicted by an emission theory.

\section{The Photoelectric Effect}

The photoelectric effect provides a striking illustration of these last two myths in the writing of an eminent experimental physicist, Robert Millikan. In the effect, electromagnetic radiation, such as light, strikes an electrode and releases cathode rays, that is, electrons. Under the wave theory of light, one would expect that the decisive determinant of the magnitude of the effect would be the intensity, that is, the energy density, of the incident light. Experimental investigations culminating in Lenard's reports of 1902 had not matched these expectations. The 
energy of the individual electrons turned out not to depend on the intensity of the incident light, but that energy was related to the frequency of the light.

In Section 8 of his "On a Heuristic Point of View about the Creation and Conversion of Light," Einstein (1905a) proposed a startling explanation of these curious features of the effect. Contrary to everything then known about light, Einstein posited that high frequency light energy does not propagate in spreading waves. Rather, the energy of this light was spatially localized into "quanta." The energy of each quanta is just hv, where h is what we now identify as Planck's constant and $v$ is the frequency of the light.

This account predicted a simple relation governing the effect:

$$
\mathrm{mv}^{2} / 2=\mathrm{V} . \mathrm{e}=\mathrm{h} v-\mathrm{p}
$$

Electrons of mass $\mathrm{m}$ are ejected with velocity $\mathrm{v}$ and kinetic energy $\mathrm{mv}^{2} / 2$. The kinetic energy is measured by applying an electrical potential that the electrons must overcome. The potential is increased in the experiments to the stopping potential $\mathrm{V}$ that just extinguishes the emitted cathode rays. It follows that the maximum kinetic energy of the individual electrons must be V.e, where $\mathrm{e}$ is the charge of the electron. In Einstein's narrative, the term hv - p results when an individual electron is energized by a single quantum of light energy hv and it loses energy $\mathrm{p}$ in working to free itself from the metallic cathode.

This relation (1) provided for a simple test, Einstein noted. The energy V.e of the released electrons, when plotted as a function of frequency $v$, should be a straight line with a slope $h$ independent of the material of the cathode.

\section{Millikan's Critique}

Robert Millikan was an unabashed critic of atomistic accounts of light emerging in the 1910s and of Einstein's light quantum quite specifically. His Millikan (1913) review was quite outspoken. After a lengthy summary of the experimental results supporting quantum behaviors, he concluded of an "atomistic theory of radiation" (Millikan, 1913, pp. 132-133; his emphasis):

Why not adopt it? Simply because no one has thus far seen any way of reconciling such a theory with the facts of diffraction and interference so completely in harmony in every particular with the old theory of ether waves. Lorenz (sic) will have nothing to do with any ether-string theory, or spotted wave-front theory, or 
electro-magnetic corpuscle theory. Planck has unqualifiedly declared against it, and Einstein gave it up, I believe, some two years ago;...

From this adversarial position, working in the Ryerson Physical Laboratory at the University of Chicago, Millikan had undertaken the arduous and delicate task of experimentally checking Einstein's equation (1). In Millikan (1916, p. 388), he reported his results:

1. Einstein's photoelectric equation has been subjected to very searching tests and it appears in every case to predict exactly the observed results.

2. Planck's h has been photoelectrically determined with a precision of about .5 per cent. and is found to have the value

$$
\mathrm{h}=6.57 \times 10^{-27} \text {. }
$$

As the title of his paper clearly conveyed--“A Direct Photoelectric Determination of Planck's ' $h$ '," Millikan restricted the success to the affirmation merely of "Einstein's photoelectric equation" and the resulting determination of h. He remained dismissive of Einstein's light quantum hypothesis. He wrote (Millikan, 1916, p. 355):

This hypothesis may well be called reckless first because an electromagnetic disturbance which remains localized in space seems a violation of the very conception of an electromagnetic disturbance, and second because it flies in the face of the thoroughly established facts of interference. The hypothesis was apparently made solely because it furnished a ready explanation of one of the most remarkable facts brought to light by recent investigations, viz., that the energy with which an electron is thrown out of a metal by ultra-violet light or X-rays is independent of the intensity of the light while it depends on its frequency.

This sharp dismissal encapsulates the two myths concerning Einstein's discovery process. First, the hypothesis is "reckless," as we would expect from a troublesome rule-breaker. Second, it was driven merely by the experiments concerning the photoelectric effect.

\section{How Einstein Discovered the Light Quantum}

We should be skeptical that contrarian rule breaking is a means of discovery. Targeting an arbitrarily chosen rule simply to be contrary most likely leads nowhere. Most rules are in no need of correction. Einstein did eventually break rules, but he had very good reasons for targeting specifically those rules he broke. He was not reckless, but measured. The impression of 
intemperate rule breaking derives from locating Einstein's researches in the context of electrodynamics and the wave theory of light. Little support for the quantum hypothesis was then available there. The locus of Einstein's project was elsewhere. It was in the thermodynamics of radiation. There Einstein found strong reasons for his light quantum hypothesis.

We have very few sources on the thinking that led Einstein to his 1905 postulation of light quantum hypothesis. Fortunately the paper itself mounts a strong case for the hypothesis. If we follow its steps we see that Einstein then already in 1905 had strong grounds for his hypothesis.

In the two opening sections, Einstein assails the security of the electromagnetic wave theory of light directly. He gives a compact demonstration that the wave theory fails badly to account for black body heat radiation. The result is now well-known, although Planck's (1900) paper has no mention of it. At thermal equilibrium, a classical radiation field in thermal equilibrium at temperature $\mathrm{T}$ has infinitely many degrees of freedom and each is excited with a mean energy of $\mathrm{kT} / 2$, where $\mathrm{k}$ is Boltzmann's constant. The total mean field energy is "infinity $\mathrm{x}$ $\mathrm{kT} / 2$," an unsustainable infinity. The demonstration was terse and devastating. It clearly showed that something was amiss in the classical theory.

What of Millikan's later concern that distinctively wave-like phenomena like interference could not be reconciled with some discrete account? In the second introductory paragraph, Einstein had already remarked that these wave-like effects are detected as time averages, which do not rule out energy localizations that are averaged away. ${ }^{11}$

The paper then turns to the principal argument for the light quantum hypothesis. From the measured thermal properties of high frequency thermal radiation, Einstein infers an expression for the entropy $S$ of a system of radiation of volume $V$ with fixed energy $E$ and high frequency $v$. It is given in relation to the entropy $\mathrm{S}_{0}$ of radiation of the same energy and frequency that occupies a different volume $\mathrm{V}_{0}$. He arrives at

$$
\mathrm{S}-\mathrm{S}_{0}=\mathrm{k}(\mathrm{E} / \mathrm{h} v) \log \left(\mathrm{V} / \mathrm{V}_{0}\right)
$$

11 Although Einstein could not then have envisaged the details, this possibility is realized in the modern quantum theory. Images of diffraction patterns in photographic film arise from the accumulated impact of many, localized photons. 
This equation merely records a fact about macroscopically accessible, thermodynamic quantities for high frequency heat radiation. It seems quite innocuous.

Einstein now shows that it is not innocuous. This equation actually encodes an unexpected microscopic constitution for high frequency heat radiation. To reveal it, he introduces what he called "Boltzmann's Principle," now commonly represented as a slogan "S = $\mathrm{k} \log \mathrm{W}$.” Its quantities require some interpretation. Over time, an isolated thermal system will fluctuate between different states. If these states can be given macroscopic equilibrium descriptions, then they can have an entropy assigned to them. The probabilities $\mathrm{W}$ and $\mathrm{W}_{0}$ of states with entropies $\mathrm{S}$ and $\mathrm{S}_{0}$ are related by

$$
\mathrm{S}-\mathrm{S}_{0}=\mathrm{k} \log \left(\mathrm{W} / \mathrm{W}_{0}\right)
$$

Next is a remark on a system of $\mathrm{n}$, spatially localized, moving points, as they move about randomly in some volume of space. Assume the motions of the points are independent of one another; it is the key assumption. Then the probability $\mathrm{W}$ that the $\mathrm{n}$ points are momentarily in some subvolume $\mathrm{V}$, in relation to the probability $\mathrm{W}_{0}$ that they are in the full volume $\mathrm{V}_{0}$, is

$$
\left(\mathrm{W} / \mathrm{W}_{0}\right)=\left(\mathrm{V} / \mathrm{V}_{0}\right)^{\mathrm{n}}
$$

Combining (3) and (4), we have

$$
\mathrm{S}-\mathrm{S}_{0}=\mathrm{k} \log \left(\mathrm{V} / \mathrm{V}_{0}\right)^{\mathrm{n}}=\mathrm{kn} \log \left(\mathrm{V} / \mathrm{V}_{0}\right)
$$

That is, a system of $\mathrm{n}$ spatially localized, independently moving points manifests thermodynamically such that its entropy $S$ varies with logarithm of the volume occupied.

The final conclusion comes from comparing (5) with the observationally derived (2) where the same logarithmic dependency appears. High frequency radiation, Einstein can conclude, behaves thermodynamically just like a system of spatially localized, independent points of energy. How much energy is in each point? One simply matches the factor (E/hv) of (2) with $\mathrm{n}$ of (5),

$$
\mathrm{E} / \mathrm{h} v=\mathrm{n},
$$

to conclude that there are $n$ quanta of energy hv comprising the total energy $E=n h v$. These last conclusions comprise precisely Einstein's hypothesis of the light quantum.

With the main work done, Einstein followed a standard mode in his papers. He completed them by showing how his main conclusion connects with experiments. It either explains past results or predicts new ones subject to test. Einstein treated three experimentally explored 
phenomena pertaining to the creation and transformation of light: photoluminescence, the photoelectric effect and gas ionization by ultraviolet light. There is no sense that the photoelectric effect has any special place in the overall paper, beyond its role as a means of testing experimentally that main proposal of the paper, derived by other means.

\section{Behind the Miraculous Argument}

The thermodynamic argument that issues in the light quantum hypothesis is so striking that I have called it the "miraculous argument." It is in my view the greatest moment of Einstein's 1905 year of miracles. The argument is so quick and so powerful in its conclusion that it easy to dismiss it as fortuitous formula juggling that just by chance happens to hit upon what later proves to be a robust and profound conclusion. This dismissal is especially easy if one has in mind the great successes of the nineteenth century wave theory. Can these successes really be reversed so easily?

However, in another context, the argument is decisive. As I have described in greater detail elsewhere (Norton 2006, 2008), while the result is revolutionary, the method used to arrive at it is a time-tested standard and one quite familiar to Einstein. The essential idea is that macroscopic thermodynamic properties of a thermal system have their microscopic constitution imprinted on them. Once one learns to read the imprinting, one can infer reliably from the macroscopic thermodynamic properties of a system to its microscopic constitution.

The venerable example extends back to the nineteenth century. It was already wellknown then that a system of $\mathrm{n}$, spatially localized, independent components exerts a pressure $\mathrm{P}$ that conforms with the ideal gas law:

$$
\mathrm{PV}=\mathrm{nkT}
$$

when confined to a volume $\mathrm{V}$ at temperature $\mathrm{T}$. To secure this law, one merely needs a system of many, spatially localized, independent components. Solute molecules in dilute solution also have this constitution. Hence their osmotic pressure P also conforms with the ideal gas law (6).

The association of the ideal gas law with this microscopic constitution was a commonplace of the later nineteenth century. It was so secure that it could be used as an analytic tool. For example, in 1887, Arrhenius used it to determine the degree of dissociation of hydrogen chloride, $\mathrm{HCl}$, in aqueous solution. If fully dissociated into hydrogen and chloride ions, the solute would have twice the number of components than the fully undissociated solute. Hence its 
osmotic pressure would also be doubled. The degree of dissociation could then be traced merely by tracking osmotic pressure.

Einstein was quite familiar with this imprinting. His first publications of 1901 and 1902 employed it. He looked at the macroscopically accessible properties of capillarity (Einstein, 1901) and the electrolytically determinable chemical potentials (Einstein, 1902). He used them in a failed attempt to establish a particular intermolecular force law. Einstein continued publishing in statistical physics from 1902 to 1904 and, finally, two of his papers of the year of miracles of 1905 depended on the technique. His doctoral dissertation (Einstein 1905b) sought to determine the size of molecules from the viscosity and diffusion properties of dilute sugar solutions. His Brownian motion paper (Einstein 1905c) sought the same from the observed motions of microscopically visible particles in suspension.

This work prepared Einstein for the light quantum. For reasons that need not detain us here, both the dissertation and the Brownian motion paper depended essentially on the simplest case of systems with a microscopic constitution of many, spatially localized, independent components. So Einstein was very familiar with the way this microscopic constitution manifests macroscopically. Hence, when this constitution manifested in the measured properties of high frequency heat radiation, Einstein was well-equipped to identify it.

Seeing the imprint of this microscopic constitution of radiation on its thermodynamics properties is not quite so straightforward. The default expectation, based on the nineteenth century experience, is to look for conformity with the ideal gas law. Heat radiation, however, seems not to conform with that law. Isotropic radiation does exert a pressure. However that pressure is determined fully by the energy density u:

$$
\mathrm{P}=\mathrm{u} / 3
$$

and the energy density $\mathrm{u}$ in heat radiation is determined fully by the temperature $\mathrm{T}$. That would seem to preclude the ideal gas law (6). Take the simplest process, an isothermal expansion. Since $\mathrm{T}$ is held constant, we have for such a process:

$$
\mathrm{P}=\mathrm{nkT} / \mathrm{V}=\text { constant } / \mathrm{V}
$$

As the gas expands the pressure will drop in inverse proportion to the volume. This elementary fact, Boyle's law, is the traditional start of the physics of gases. If we expand a system of heat radiation isothermally, however, the energy density remains fixed and thus so does the pressure. This seems definite: the ideal gas law fails for heat radiation. 
The key to seeing that the law does not fail lies in a disanalogy between ideal gases and heat radiation. The number of component molecules $\mathrm{n}$ in an ideal gas is fixed. The number of quanta $\mathrm{n}$ in high frequency heat radiation is highly variable. When a volume of heat radiation expands at constant temperature, its total energy increases in proportion to its volume and, as a result, the number of quanta increases in proportion to its volume. ${ }^{12}$ This restores conformity with the ideal gas law. An isothermal expansion of a volume of heat radiation is described by

$$
\mathrm{P}=\mathrm{nkT} / \mathrm{V}=(\mathrm{n} / \mathrm{V}) \mathrm{kT}=\mathrm{constant}
$$

since each of $(\mathrm{n} / \mathrm{V})$ and $\mathrm{kT}$ are constants in an isothermal expansion.

While this restores the compatibility of the properties of heat radiation with the ideal gas law, the macroscopic relation " $\mathrm{p}=$ constant" for an isothermal expansion is a poor means of revealing the microscopic constitution of heat radiation. Einstein needed a more convincing indicator of the constitution. He found it by the expedient of suppressing the confounding disanalogy between ideal gases and heat radiation: the variability of the number of component quanta. Einstein's relation (2) presumes that the total energy $\mathrm{E}$ and frequency $v$ of the radiative system is the same in the two states related by it. There are very few processes involving systems of radiation that maintain both constant. A random fluctuation in an isolated system between volumes is one of them. For such a process, Einstein's relation (2) obtains.

In the fluctuation process, the entropy of the heat radiation $S$ varies with the logarithm of the volume V. This dependency, Einstein showed elsewhere in 1905, is a manifestation of a system consisting of many, spatially localized, independent components. This result was included within the second section of his Brownian motion paper (Einstein, 1905c). Using the standard methods developed in his earlier papers, Einstein showed that the entropy of such a system is given by (up to an additive constant in temperature)

$$
\mathrm{S}=\mathrm{E}(\mathrm{T}) / \mathrm{T}+\mathrm{nk} \log \mathrm{V}
$$

where the energy $\mathrm{E}(\mathrm{T})$ varies with $\mathrm{T}$ but not $\mathrm{V}$. He then proceeded to show that this logarithmic dependency of entropy on volume leads directly to the ideal gas law.

At this point, the one might imagine that Einstein's work is complete. That the entropy of high frequency radiation varies with the logarithm of volume (2) is recovered from macroscopic

12 For the case of high frequency radiation, Einstein $(1905, \S 5)$ computes the mean energy of each quanta as $3 \mathrm{kT}$. Thus the total number of quanta $\mathrm{n}=\mathrm{E} / 3 \mathrm{kT}=(\mathrm{u} / 3 \mathrm{kT}) \mathrm{V}$, since $\mathrm{E}=\mathrm{uV}$. 
measurements. Through (7), we know that this logarithmic dependency is characteristic of systems of many, spatially localized, independent components. And Einstein has shown that this dependency leads directly to the familiar ideal gas law, the traditional signature of systems with this microscopic constitution.

The trouble is that the connection between the measurement based properties of radiation (2) and the theoretically derived (7) is not close enough. In deriving (7) Einstein had employed a formalism in which the number of components is fixed at the outset. There one begins with a phase space whose dimension is fixed at the start by the number of components. The number of quanta is variable; the dimensions of the phase space is not.

Einstein's solution to this last problem is a foundational tour de force. The basic result needed is that, in systems of many, spatially localized, independent components, the entropy varies with the logarithm of volume. He rederived this result with essentially none of the encumbrances of the then standard statistical mechanical formalism. In the space of just two and a half journal pages, he not only introduces "Boltzmann's Principle" (3) for the first time by name in the literature, he also gives a compact demonstration of it. Since the probabilities "W" of independent systems multiply and their entropies " $\mathrm{S}$ " add, the only possible relation between them is " $\mathrm{S}=\mathrm{k} \log \mathrm{W}$." As we have seen, he applies this principle to systems of many, spatially localized, independent components and recovers the entropy-volume relation (5). From it, he then derives the ideal gas law. He could then apply (5) to the observationally grounded (2) and complete the recovery of the light quantum hypothesis.

Einstein has had to assume very little in arriving at these results. He was quite aware of this distinctive feature. In a paper that is quite terse in relation to the profound significance of its results, he remarks immediately after (5) is derived:

It is noteworthy that the derivation of [equation (5)] requires the making of no assumption about the law according to which the molecules move.

This is the important point, for in 1905, Einstein could presume very little about the properties of his light quanta. Their dynamics, it would seem, could hardly fit into the familiar mold of the Hamiltonian mechanics of particle then applied to gases. The quanta pop in and out of existence, when one expands or contracts the space holding them. 


\section{Overestimated Importance}

The mistaken idea that Einstein proposed the light quantum principally to account for the photoelectric effect is quite widespread. The move from the empirically confirmed equation (1) governing the effect to the notion of the light quantum is pedagogically fast and easy; and it is pervasive in quantum theory textbooks. A hasty pedagogue can easily elevate this pedagogic pathway to the pathway of Einstein's discovery. In a recent example, (Grynberg et al., 2010, p. 179; emphasis in original) write:

The existence of a threshold frequency for the incident radiation, $\omega_{\mathrm{S}}$, below which the emission of electrons does not occur is a characteristic of the photoelectric effect that classical physics, or, more precisely, classical electrodynamics, is unable to account for. It is this feature that led Einstein, in 1905, to postulate that monochromatic light of frequency $\omega$ is composed of particles, later to be known as photons...

Another contributing confusion is a lack of clarity over just how much had been done by Planck prior to 1905. As Kuhn (1978) controversially urged, it was far from clear in 1900 to Planck or others in 1900 that a revolutionary shift in physics had just begun. Einstein himself was unclear that his 1905 light quantum hypothesis had some strong affinities with Planck's work of 1900. He acknowledged in 1906 that he only then saw that "Planck's theory makes implicit use of the aforementioned hypothesis of light quanta." (Einstein, 1906, p. 199)

Yet repeatedly we find it reported that Einstein's discovery is merely the application of Planck's theory to the photoelectric effect. It is how Millikan glosses Einstein's discovery in Millikan's Nobel Prize lecture:

... in 1905 by Einstein who, by combining [Thomson's corpuscular theory of light] with the facts of quanta discovered by Planck ${ }^{[13]}$ through his analysis of black-body radiation, obtained an equation which should govern, from his viewpoint, the interchange of energy between ether waves and electrons...

Shamos (1959, p. 233), in his editorial introduction, notes:

13 Millikan's footnote is to Planck (1900). 
It was at this point, in 1905, that Einstein showed how Planck's new quantum

theory of radiation could be used to account for the photoelectric effect.

Finally Einstein's 1921 Nobel Prize citation read "for his services to Theoretical Physics, and especially for his discovery of the law of the photoelectric effect". It singled out his "law of the photoelectric effect," suppressing mention of the proposal of the light quantum that engendered it.

Einstein's proposal of the light quantum was, in his own estimate, revolutionary. However it was not an exercise in contrarian rule breaking based only on the slender support of one phenomenon, the photoelectric effect.

\section{General Relativity}

Einstein was prodigious in his discoveries. Our enduring interest in them has ensured an equally prodigious supply of oversimplified accounts of what powered them, as the few examples discussed here illustrate. These examples all arise through hasty oversimplification by later commentators. In one notable case, Einstein himself was also responsible for a confusing narrative of discovery. In 1905, the special theory of relativity had re-established the relativity of inertial motion in physics. In 1907 Einstein set upon the further task of extending this relativity to accelerated motion. Eight years later, an exhausted Einstein declared success. The general theory of relativity was completed.

The discovery of the theory by Einstein, working essentially alone, is universally acclaimed as an extraordinary achievement, likely without peer in all of 20th century science. On Einstein's account, the discovery was driven by the goal of generalizing the relativity of motion. That goal was the star by which he navigated. The difficulty is that there is now a growing consensus that Einstein's theory does not generalize the relativity of motion to acceleration. That presents a serious problem for people like me, who believe that great discoveries in science are grounded in rational exploration. How could Einstein successfully cross a treacherous ocean using defective navigation guides? How can we explain the success?

The goal of generalizing the relativity of motion was just one of many ideas that Einstein sought to incorporate into his developing theory. We can, I believe, already explain his success by Einstein's accommodation of just a few of these; and they do not include the extension of the 
relativity of motion to acceleration. Just which these should be is something open to debate. ${ }^{14}$ For an entry into this literature, see Norton (1993) and Janssen (2014).

\section{Conclusion: How to Discover Like Einstein}

The myths that surround Einstein's pathway to his discoveries are persistent and tenacious. This fact derives, I believe, from a simple mechanism. We are all too ready to read our own interests, aspirations, obsessions and prejudices into Einstein's discoveries. His operational analysis of simultaneity became one of the most prominent conceptual analyses of the twentieth century. Our later fascination with it inclines us to believe that it must have played a crucial role in Einstein's discovery. We find our pedagogy greatly simplified if we draw a line for our students straight from one puzzling experiment to Einstein's proposal. So it is easy for us to imagine that this line is the path that Einstein walked. We might like to believe that great discoveries can be made with the innocence of childlike thinking or by the contrarian breaking of rules. So we are ready to believe that Einstein was a naïf or contrarian. Finally, if we are unsympathetic to Einstein's discoveries, it is tempting to portray him making over hasty generalizations from one experiment in the interests of intemperate rule breaking.

What does it take to discover like Einstein? A closer look at Einstein's work gives us a list that should have been obvious at the outset. It is easy to write down, but it does not provide a simple recipe that can turn each of us into an Einstein. To discover like Einstein:

Be very smart and creative. There is, as far as I can see, no substitute for raw aptitude.

Master all the science that comes before you. Einstein could do more than those who came before him precisely because he had absorbed their work and understood it fully. Only then could he see their failings and know just where the new theories are to be found.

14 My choices: on the basis of his principle of equivalence, Einstein decided at the outset in 1907 that gravitational processes were to be incorporated into the theory by means of alterations to the structure of space and time. With that, one is led almost uniquely to general relativity by the further requirements of the Newtonian limit, that energy-momentum is to be conserved and that gravitational energy is a source of gravity. 
Work very hard and tolerate many setbacks. Einstein's ascent was arduous with many dead ends and disappointments. Yet Einstein remained steadfast in his pursuit and, as progress became harder, he would consider more extreme ideas if they showed promise.

Enjoy the fleeting moments of transcendent insight these efforts bring. They will come, but they are not assured. Einstein was never satisfied with his account of the light quantum. He lamented to his friend and confidant Michele Besso in 1951 "All these fifty years of pondering have not brought me any closer to answering the question, What are light quanta?" 15

Be brilliant at presenting complex ideas simply. Then your readers will marvel at how straightforward the discovery proved to be and believe that it was so simple a child could have done it.

Be just a little bit lucky: your aptitudes must match the problem to be solved. Einstein's powerful physical intuition was just what was needed to lead to special relativity and the light quantum. Things changed with the elaboration of quantum theory. Then Niels Bohr's affection for odd thought and logical contradiction proved fertile, for a while. Einstein's nineteenth century grounding 16 proved to be an obstacle.

\section{References}

Bridgman, Percy W. (1927) The Logic of Modern Physics. New York: MacMillan.

Calaprice, Alice (ed.) (2011) The Ultimate Quotable Einstein. Princeton: Princeton University Press.

Einstein, Albert (1901) "Folgerungen aus dem Capillaritätserscheinungen," Annalen der Physik, 4, pp. 513-523.

Einstein, Albert (1902) "Ueber die thermodynamische Theorie der Potentialdifferenz zwischen Metallen and vollständig dissocierten Lösungen ihre Salze and über eine elektrische Methode zur Erforschung der Molecularkräfte,” Annalen der Physik, 8(1902), pp. 798814.

15 As quoted in Pais (1982, p. 382).

16 As described in Norton (2012). 
Einstein, Albert (1905) “Zur Electrodynamik bewegter Körper," Annalen der Physik, 17, pp. 891-921. Translated as "On the Electrodynamics of Moving Bodies." pp. 35-65 in H. A. Lorentz, et al., The Principle of Relativity. Methuen, 1923; repr. New York: Dover, 1952. Einstein, Albert (1905a) "Über einen die Erzeugung and Verwandlung des Lichtes betreffenden heuristischen Gesichtspunkt," Annalen der Physik, 17, pp. 132-148.

Einstein, A. (1905b). Eine neue Bestimmung der Moleküldimensionen. Bern: Buchdruckerei K. J. Wyss; also in Annalen der Physik, 19(1906), 289-305.

Einstein, A. (1905c). "Über die von der molekularkinetischen Theorie der Wärme geforderte Bewegung von in ruhenden Flüssigkeiten suspendierten Teilchen," Annalen der Physik, $17,549-560$.

Einstein, Albert (1906) “Zur Theorie der Lichterzeugung and Licht Ausbreitung," Annalen der Physik, 20, pp. 199-206.

Einstein, Albert (1907) “Über das Relativitätsprinzip und die ausdemselben gezogenen Folgerungen," Jahrbuch der Radioaktivität und Elektronik, 4, pp. 411-62.

Einstein, Albert (1917) Relativity: The Special and the General Theory. A Popular Exposition. Braunschweig: Friedr. Vieweg \& Sohn. Trans. R. W. Lawson. London: Methuen \& Co. 1920.

Einstein, Albert (1949), "Autobiographical Notes," pp. 2-95 in, P. A. Schilpp, ed., Albert Einstein-Philosopher Scientist. 2nd ed. New York: Tudor Publishing, 1951, pp. 671-72. Reprinted with a correction as Autobiographical Notes. La Salle and Chicago: Open court, 1979.

Einstein, Albert (1956) “Autobiographische Skizze,” pp. 9-17 in Seelig, Carl (ed.) Helle ZeitDunkle Zeit. Zurich: Europa Verlag.

Fölsing, Albrecht (1997) Albert Einstein: A Biography. Trans. Ewald Osers. New York: Viking. Frank, Philipp (1947) Einstein: His Life and Times. Trans. George Rosen. Ed. and rev. Shuichi Kusaka. New York: Alfred A. Knopf.

Allan Franklin (forthcoming) "Physics Textbooks Don't Always Tell the Truth.” Physics in Perspective.

Galison, Peter (2003) Einstein's Clocks, Poincaré's Maps: Empires of Time. New York: W. W. Norton \& Co. 
Gardner, Howard (20111) Frames of Mind: The Theory of Multiple Intelligences. New York: Basic.

Grynberg, Gilbert; Aspect, Alain; and Fabre, Claude (2010) Introduction to Quantum Optics. Cambridge: Cambridge University Press.

Holton, Gerald (1969) “Einstein, Michelson, and the 'Crucial' Experiment," Isis, 60, pp. 132197.

Janssen, Michel (2014) “'No Success Like Failure ...': Einstein’s Quest for General Relativity, 1907-1920," Ch. 6 in Janssen and Lehner (2014).

Janssen, Michel and Lehner, Christoph, eds., (2014) Cambridge Companion to Einstein. Cambridge: Cambridge University Press.

Kamlah, Andreas (1994) "The Problem of Operational Definitions," Ch. 6 in W. Salmon and G. Wolters, eds., Logic, Language, and the Structure of Scientific Theories. Pittsburgh: University of Pittsburgh Press.

Kuhn, Thomas S. (1978) Black-Body Theory and the Quantum Discontinuity. Oxford: Clarendon Press.

Levenson, Thomas and MacDonald, Ross (2004) “Einstein's Gift for Simplicity," Discover, September 30, 2004 http://discovermagazine.com/2004/sep/einsteins-gift-for-simplicity

Loehle, Craig (2010) Becoming a Successful Scientist: Strategic Thinking for Scientific Discovery. Cambridge: Cambridge University Press.

Madden, Kendall (2005) "Physicist and historian Peter Galison to deliver Hofstadter Lecture on relativity," Stanford Report, May 16, 2005. http://news.stanford.edu/news/2005/may18/hofstadter-051805.html

Martinez, Alberto A. (2004), "Material History and Imaginary Clocks: Poincare', Einstein, and Galison on Simultaneity, Physic in Perspective, 6, pp. 224-240.

Millikan, Robert (1913) “Atomic Theories of Radiation,” 37, No. 943 (Jan. 24, 1913), pp. 119133.

Millikan, Robert (1916) “A Direct Photoelectric Determination of Planck's 'h', Physical Review, 7, pp. 355-388.

Mook, Delo E. and Vargish, Thomas (1987) Inside Relativity. Princeton: Princeton University Press. 
Norton, John D. (1993) "General Covariance and the Foundations of General Relativity: Eight Decades of Dispute," Reports on Progress in Physics, 56, pp.791-858.

Norton, John D. (2004) "Einstein's Investigations of Galilean Covariant Electrodynamics prior to 1905," Archive for History of Exact Sciences, 59, pp. 45-105.

Norton, John D. (2006)"Atoms Entropy Quanta: Einstein's Miraculous Argument of 1905," Studies in History and Philosophy of Modern Physics, 37 (2006), pp. 71-100.

Norton, John D. (2008) "Einstein's Miraculous Argument of 1905: The Thermodynamic Grounding of Light Quanta" in C. Joas, C. Lehner and J. Renn (eds.), HQ1: Conference on the History of Quantum Physics: Max Planck Institute for the History of Science. Preprint 350. Vol. 1, pp. 63-78.

Norton, John D. (2012) "Einstein as the Greatest of the Nineteenth Century Physicists," pp. 14251 in Proceedings, Seventh Quadrennial Fellows Conference of the Center for Philosophy of Science (12-14 June 2012; Mugla, Turkey). http://philsciarchive.pitt.edu/9135/

Norton, John D. (2013) "Chasing the Light: Einstein's Most Famous Thought Experiment," Thought Experiments in Philosophy, Science and the Arts, eds., James Robert Brown, Mélanie Frappier and Letitia Meynell, New York: Routledge. pp. 123-140.

Pais, Abraham (1982) "Subtle is the Lord..." The Science and the Life of Albert Einstein. Oxford: Oxford University Press.

Planck, Max (1900) “Zur Theorie des Gesetzes der Energieverteilung im Normalspectrum” Verahndlungen der Deutschen physikalischen Gesellschaft, 2, pp. 237-45. (Presented, December 14, 1900.)

Reichenbach, Hans (1957) Philosophy of Space and Time. New York:Dover.

Seelig, Carl (1956) Albert Einstein: A Documentary Biography. Trans. Mervyn Savill. London: Staples Press.

Shamos, Morris H. (ed.) (1959) Great Experiments in Physics: Firsthand Accounts from Galileo to Einstein. New York: Holt, Rinehart and Winston; repr. New York: Dover, 1987.

Stachel, John et al. (eds.) (1987) The Collected Papers of Albert Einstein: Volume 1: The Early Years: 1879-1902. Princeton: Princeton University Press.

Thorpe, Scott (2015) How to Think Like Einstein: Simple Ways to Break the Rules and Discover your Hidden Genius. Naperville, IL: Sourcebooks. 
Wertheimer, Max (1959) Productive Thinking. Enlarged ed., New York: Harper \& Bros.

Wheeler, John A. (1980), “Albert Einstein 1879-1955: A Biographical Memoir,” National Academy of Sciences. Washington, DC, 1980.

http://www.nasonline.org/publications/biographical-memoirs/memoir-pdfs/einsteinalbert.pdf 